\title{
The role of bars
}

\author{
F. Combes ${ }^{1}$ \\ ${ }^{1}$ Observatoire de Paris, LERMA, 61 Av. de l'Observatoire, F-75014, Paris, France \\ email:francoise.combes@obspm.fr
}

\begin{abstract}
Secular evolution and fueling is driven by bars in spiral galaxies, and the related dynamical processes are reviewed. It is shown that gravity torques dominate over viscous torques, and produce gas infall to the center. In this infall, the bar wave accepts the angular momentum, which produces its destruction. In the end, a central mass concentration is built, which also contributes to this destruction. While gas can be stalled at ILR for a while, secondary bars then take over the fueling. In a galaxy life-time, several bar episodes can successively develop. Through external gas accretion, disks are replenished by intermittence, between two bar phases. While the general trend for a galaxy is to evolve towards early-types, its morphology can oscillate, and turn back transiently to later-types.
\end{abstract}

\section{The problem of bars and AGN fueling}

Disks of spiral galaxies can be considered as accretion disks: matter tends to concentrate to reach the least energy state, but the angular momentum has to be transfered outwards. Since viscous torques are not sufficient over a Hubble time, gravity torques play the main role, through formation of bars and spirals. Numerical simulations reveal that bars are very efficient to drive the gas of a galaxy inwards, however there is no clear correlation between nuclear activity and bars in the observations (e.g. Mulchaey \& Regan 1997). There could even be a lower fraction of strong bars in Seyferts (Shlosman et al 2000). The only positive signs of the bar action is the observation of more outer rings in Seyfert galaxies (Hunt \& Malkan 1999, and Agüero et al, this meeting), and more gas concentration in barred galaxies (Sakamoto et al 1999). Also, there is always circumnuclear dust in AGN, while not always in normal galaxies (Martini et al 2003).

The main reason for this lack of correlation could be the different time-scales: the duty cycle for nuclear activity is of the order of $40 \mathrm{Myr}$, much shorter than the dynamical time-scales of gas inflow (at least $300 \mathrm{Myr}$ ). The case of galaxy interactions is similar, since internal bars are then also the motor of gas inflow. Nuclear starbursts are often simultaneously observed with nuclear activity (cf Maia, Lim et al, Storchi-Bergman et al, this meeting).

\section{Gas flows in barred galaxies}

Gas behaviour in a barred potential has been studied for a long time (e.g. Sanders \& Huntley 1976), but the larger spatial resolution reached recently has allowed to better estimate the influence of viscosity (Maciejewski et al 2002). Already Englmaier \& Gerhard (1997) revealed the large influence of the sound speed in the gas behaviour. The larger the velocity dispersion in the gas, the larger the mean free path of the particles, and the larger the distance over which they can exchange angular momentum, so that shear is more important. With large velocity dispersion, the gas that was previously trapped at ILR can further fall inwards (Maciejewski et al 2002, cf also Ann, this meeting). The role of bar characteristics (strength, axis ratio, etc..) on gas inflow has been examined in 
details (Regan \& Teuben 2004), weak bars have only marginal effect, while gas velocity dispersion or sound speed Vs is a key parameter. Bars drive the gas until the ILR only, if $\mathrm{Vs}$ is small. If $\mathrm{Vs}$ is larger than $20 \mathrm{~km} \mathrm{~s}^{-1}$, the viscous shear can drive the gas inwards.

\subsection{Influence of gas physics}

The multi-phase interstellar medium is frequently over-simplified, and simulated in 2D. Wada \& Norman (2001) have shown part of the hidden conplexity in detailed 3D gas simulations in a fixed spherical potential (with dark matter, stars, central black hole). SN explosions are followed to heat the gas, whose scale height increases with the square root of star formation rate. Even with large viscosity and velocity dispersion, the gas flow rate towards the center is low $\left(<1 \mathrm{M}_{\odot} / \mathrm{yr}\right)$. However, there is no bar in these models.

The fact that viscous torques do not predominate in galaxy disks is supported by the observations of resonant rings, in particular the outer rings, due to the presence of positive gravity torques between corotation and OLR (Schwarz 1981). Also the large frequency of nuclear rings, where gas accumulates and form stars (Buta \& Combes 1996, 2000) gives the idea of a low sound speed of gas, with low velocity dispersion.

\subsection{Gravity versus viscous torques}

In numerical simulations, several measurements can be done to test the importance of viscous relative to gravity torques. When there is no bar, the gas is not inflowing, which means that viscous torques alone are insufficient. But they could play a role when there is a bar, since orbits are no longer circular, and the shear could then be higher. It is the gas dissipation that provokes the phase shift between gas and stars, and allows gravity torques to play a larger role. In models where gas is represented by sticky particles, it is straightforward to compute the angular momentum exchanged in cloud collisions, and compare to the gravity torques. Such a comparison (Bournaud \& Combes 2004) is displayed in Figure 1, where it is clear that the inflowing gas loses its angular momentum only by gravity torques.

Measurements of gravity torques from the observations confirm that the importance of gas inflow is not over-estimated in the simulations; the phase shift of the characteristic dust-lanes, offset towards the leading edge of the bar, is of the same magnitude, and the torques derived from the red images are of the same order as the computed ones (e.g. Block et al 2002).

\section{Destruction of bars}

The consequence of the gas inflow towards the center is the formation of a central mass concentration (CMC) and the destruction of the bar. This was shown both by Nbody simulations, and orbit computations in fixed potentials (Hasan et al 1990, 1993, Combes 1994, Norman et al 1996, Berentzen et al 1998). Bars are destroyed by 1-5\% mass concentrations within $1 \mathrm{kpc}$ (percentage with respect to the disk mass). According to the hardness of the CMC, orbits sustaining the bar (x1) are scattered by a black hole, and become chaotic, or bars are weakened or dissolved, by a concentrated bulge or nuclear

disk. Destruction is more efficient for a more concentrated CMC (a true black hole of $0.5 \%$ mass is sufficient, Hozumi \& Hernquist 1999) and also if the growth-time of the $\mathrm{CMC}$ is short.

Recently Shen \& Sellwood (2004) contest the fragility of bars, since usual black hole masses in spiral galaxies are not large enough, and softer gas concentrations only weaken the bars. They use however a non self-consistent galaxy model, with an artificial CMC 


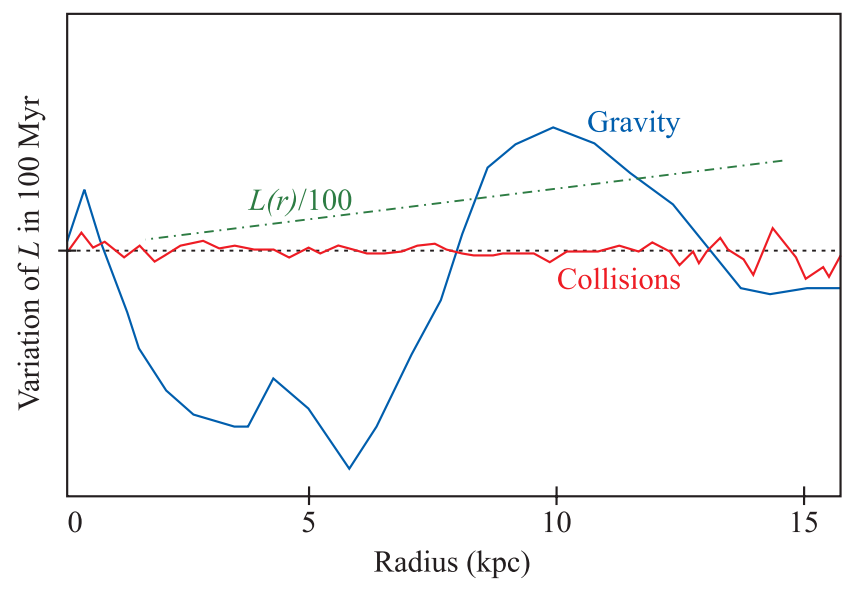

Figure 1. Comparison of the angular momentum lost in cloud collisions (viscous torques), and that due to gravity torques (from Bournaud \& Combes 2004). Gravity torques change sign at corotation.

growth in the center, without gas. They do not consider how the CMC is formed, which is in itself the main destruction mechanism.

\subsection{Role of gas in bar destruction}

One essential factor in the bar destruction, that was overlooked until now, is the angular momentum loss of the infalling gas to the bar wave (Bournaud \& Combes 2004). Gas is driven in by the bar gravity torques, and its angular momentum is taken up by the bar wave. Since the bar, inside its own corotation, has negative angular momentum, this destroys the bar (the loss of angular momentum from the gas infalling from corotation to center is of the same order).

Figure 2 compares several N-body experiments with gas and star formation, to probe the role of various phenomena. The gas loss of angular momentum is sufficient to destroy the bar completely, and this occurs even before a CMC is built. The presence of a CMC of only $1 \%$ is not enough to destroy the bar, but $1-2 \%$ of gas infall is enough, which explains the result of previous simulations (Berentzen et al 1998, Bournaud \& Combes 2002, 2004).

Since the main reason of bar destruction is not the presence of a CMC, it will then be easier to re-form a bar later.

\subsection{Double bars and gas inflow}

Nested bars are found in N-body simulations to decouple from the primary bar when 2 ILR exist, and consequently perpendicular x 2 orbits. The nuclear and primary bars have one resonance in common, which avoids the chaos, and allows the coupling and exchange of energy (Friedli \& Martinet 93).

The presence of this second bar, which torques prolonge that of the primary bar, is essential for gas inflow. Without it, the gas may be stalled at ILR. The only way to drive it to the center is through viscous torques, in the case of strong shocks and large velocity dispersion (Maciejewski 2002). Another possibility, if the gas accumulates and is cold, is clump instability; giant clouds will lose angular momentum through dynamical friction against the bulge. 


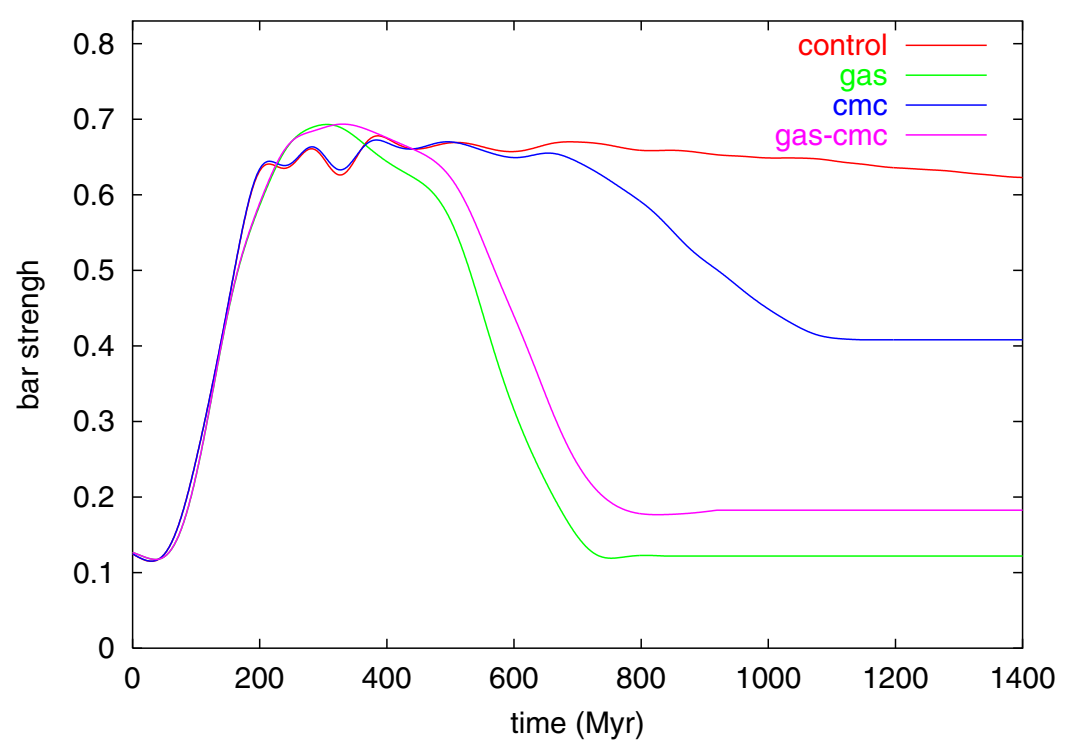

Figure 2. Strength of the bar as a function of time, for the control run at the top (stellar disk without gas), for the realistic model (light curve at bottom), and the same but where the CMC is progressively removed (full curve, "gas-cmc", almost coincident). When only an equivalent CMC is introduced, the bar is only weakened (intermediate curve). The model galaxy had $6 \%$ of mass in gas with respect to the disk, a bulge of $25 \%$, and $1 \%$ of disk mass in gas has moved inside $300 \mathrm{pc}$ at the end.

\section{Bar reformation}

Since bars in spiral galaxies with gas are easy to destroy, they must be regularly reformed, to explain the observed bar frequency today. The typical destruction timescale is $1 \mathrm{Gyr}$, as is the formation time-scale.

\subsection{Statistics on bar strength}

It is commonly adopted from galaxy classification that about two thirds of galaxies are barred ( $\mathrm{SB}$ and $\mathrm{SAB}$ ), while one third is strongly barred (SB). This percentage corresponds to visible bands, there are even more barred galaxies in near-infrared images. To quantify better bar strength in spiral galaxies, and compare to N-body models, we have undertaken to compute the parameter $Q_{b}$ for the near-infrared OSU survey of 163 galaxies (Eskridge et al 2002).

The bar strength parameter $Q_{b}$ is estimated from the Fourier decomposition of the potential, it is the ratio of the $m=2$ component of the tangential force to the radial force. The potential is estimated from the NIR images after disk deprojection, and assuming a constant M/L (Block et al 2002). The main result is a dearth of axi-symmetric galaxies, and a large number of strongly barred galaxies (see also Whyte et al 2002, whose estimation relies on bar axis ratio).

This observed distribution of bar strength cannot be accounted for in numerical models of isolated galaxies. After a few Gyr, galaxies become axisymmetric, or weakly barred. The only possibility to reform the bar is gas accretion from outside, to replenish the disk, decrease the bulge-to-disk mass ratio, and make the disk unstable again. Numerical simulations can quantifify more precisely the accretion rate, by confrontation to the observed bar strength distribution. 


\subsection{Numerical simulations}

Since accretion of large quantities of gas have to be considered, star formation is essential to follow the fate of this gas. Simulations will show that the gas fraction remains almost constant during evolution, the rate of gas accretion being comparable to the rate of star formation (roughly $10 \mathrm{M}_{\odot} / \mathrm{yr}$ ).

Also, the required gas could come from the mass loss from stars, and it is important to take into account the mass loss quantitatively, via a continuous recycling, and not instantaneous, scheme (e.g. Jungwiert, Combes \& Palous 2001).

Gas accretion simulations have been carried out with a 3D FFT N-body code, and a sticky particles model for gas clouds (Bournaud \& Combes 2002). Star formation is followed according to a Schmidt law recipe, with exponent 1.4. The morphology of simulated spiral galaxies change considerably in the long term when gas accretion is taken into account. An isolated galaxy first forms a bar, and the gas is driven inwards. The morphology corresponds to a normal spiral, but only for 1 Gyr. Then the bar is destroyed, and the galaxy turns axisymmetric. With accretion, the galaxy is re-juvenated: the disk becomes unstable again to bars and spirals. Long term evolution requires gas accretion, in order to obtain the presently observed spiral morphologies.

Since the bar exerts positive torques on the gas from corotation until OLR, the accreted gas has to wait for the destruction of the bar to replenish the disk. During a galaxy life-time, gas accretes by intermittence. First it is confined outside OLR, until the bar weakens, through gas infall from corotation to the center. Then the galaxy becomes axisymmetric, and only through viscosity the external gas settles in the disk, to make it unstable again to bar formation.

Given the time-scales to bar destruction and formation, it is possible to expect 3 or 4 bar episodes in a galaxy life-time. Simulations show that the successive bar pattern speeds increase with time (the corotation radius shrinks). It is interesting to note that, in this evolution, the bulge-to-disk mass ratio can decrease, and therefore the galaxy can evolve towards later types, for a short period. While the usual trend is to evolve towards early-types, the morphological type of a spiral galaxy can oscillate.

\subsection{Origin of the gas}

Numerical simulations with accretion predict the bar frequency today, as a function of the accretion rate. When compared to the observed frequency of bars (see above), a large accretion rate is required, such that a galaxy doubles its mass in 10 Gyr. For a galaxy of the Milky Way type, this corresponds to an accretion rate of the order of $10 \mathrm{M}_{\odot} / \mathrm{yr}$.

Could this gas be accreted through galaxy interactions? Massive galaxy interactions and mergers do not develop disks but spheroids. Gas-rich dwarf companions cannot provide more than $10 \%$ of the accretion, since those interactions heat the galaxy disk (e.g. Toth \& Ostriker 1992). What is required is a continuous source of cold gas, which could come from the cosmic filaments in the near environment of galaxies.

Cosmological accretion is comparable to what is required for bar reformation. This accretion is compatible with doubling the mass in 10 Gyr. It is able also to explain the history of star formation in typical spiral galaxies (Semelin \& Combes 2002). The accretion is intermittent, rythmed by gravitational instabilities; the gas infalls also fuels the central black hole, which grows in parallel to the bulge (cf Combes 2000).

\section{Conclusions: fueling processes}

When gravity torques exist, they are the most efficient mechanism to fuel gas towards the center. The unstable disk forms first a primary bar, then a secondary bar decouples, 
but is more short-lived: ILR can stop inflow only for a while. When the gravity torques disappear, then: - mild gravitational instabilities develp velocity dispersion and viscosity

- or create clumps of gas; the disk then very non-axisymmetric produces inflows

- or dynamical friction of giant clouds against the bulge brings the gas to the center,

- alternatively $m=1$ asymmetries, due to a companion, or anisotropic accretion, accelerate infall (examples in NUGA survey, cf Garcia-Burillo, this meeting).

Numerical simulations have clearly shown how all these dynamical processes are selfregulated. It is unrealistic to study phenomena independently, like gas flow in rigid bars for instance, since the gas infall regulates the bar strength. Fueling bars self-destroy.

Galaxies are not closed systems, they accrete gas by intermittence, and double their mass every 10 Gyr. This accretion cannot come mainly from companions, but come from gas in the near cosmic filaments. This high accretion rate is required to explain bar/spiral reformation, and also star formation.

\section{References}

Berentzen, I., Heller, C. H., Shlosman, I., \& Fricke, K. J. 1998, MNRAS, 300, 49

Block, D., Bournaud, F., Combes, F., Puerari, I., \& Buta, R. 2002, A\&A, 394, L35

Bournaud, F., \& Combes, F. 2002, A\&A, 392, 83

Bournaud, F., \& Combes, F. 2004, A\&A Letter, in press

Buta, R., \& Combes, F. 2000, in Dynamics of Galaxies from the Early Universe to the Present, (eds. F. Combes, G. A. Mamon and V. Charmandaris), ASP Conf Series, 197, 11

Buta, R., \& Combes, F. 1996, Fund. Cosmic Phys., 17, 95

Combes, F. 2000, in Dynamics of Galaxies from the Early Universe to the Present, (eds. F. Combes, G. A. Mamon and V. Charmandaris), ASP Conf Series, 197, 15

Combes, F. 1994, in Mass-transfer induced activity in galaxies, (ed. I. Shlosman), Cambridge Univ. Press., 170

Englmaier, P., \& Gerhard, O. 1997, MNRAS, 287, 57

Eskridge, P. B., et al., 2000, AJ, 119, 536

Friedli, D., \& Martinet, L. 1993, A\&A, 277, 27

Hasan, H., \& Norman, C. A. 1990, ApJ, 361, 69

Hasan, H., Pfenniger, D., \& Norman, C. 1993, ApJ, 409, 91

Hozumi, S., \& Hernquist, L. 1999, in Galaxy Dynamics, (eds. D. R. Merritt, M. Valluri, and J. A. Sellwood), ASP Conf Series, 182, 259

Hunt, L., \& Malkan, M. 1999, ApJ, 516, 660

Jungwiert, B., Combes, F., \& Palouš, J. 2001, A\&A, 376, 85

Maciejewski, W., Teuben, P., Sparke, L., \& Stone, J. 2002, MNRAS, 329, 502

Martini, P., Regan, M. W., Mulchaey, J. S., \& Pogge, R. W. 2003, ApJ, 589, 774

Mulchaey, J. S., \& Regan, M. W. 1997, ApJ, 482, L135

Norman, C., Sellwood, J., \& Hasan, H. 1996, ApJ, 462, 114

Regan, M., \& Teuben, P. 2004, ApJ, 600, 595

Sakamoto, K., Okumura, S. K., Ishizuki, S., \& Scoville, N. Z. 1999, ApJ, 525, 691

Sanders, R., \& Huntley, J. 1976, ApJ, 209, 53

Schwarz, M. P. 1981, ApJ, 247, 77

Semelin, B., \& Combes, F. 2002, A\&A, 388, 826

Shen, J., \& Sellwood, J. 2004, ApJ, 604, 614

Shlosman, I., Peletier, R., \& Knapen, J. 2000, ApJ, 535, L83

Toth, G., \& Ostriker, J. P. 1992, ApJ, 389, 5

Wada, K., \& Norman, C. 2001, ApJ, 547, 172

Whyte, L. F., et al. 2002, MNRAS, 336, 1281 